\title{
Search for the $B$-meson decay to four baryons
} $B \rightarrow p p \bar{p} \bar{p}$

\author{
Laura Zani ${ }^{* \dagger}$ \\ INFN - National Institute for Nuclear Physics \\ E-mail: laura.zani@pi.infn.it
}

Final states containing baryons are kinematically accessible to weakly decaying $B$ mesons. The measurement of the exclusive branching fractions of baryonic $B$ decays, as well as studies on the dynamic of the decay, may allow better understanding of baryon production in $B$ decays and, more generally, hadron fragmentation into baryons. We present here a search for the decay $B \rightarrow p p \bar{p} \bar{p}$, not yet observed, by using a data set consisting of about 470 million $B \bar{B}$ pairs collected with the BABAR detector at the SLAC National Accelerator Laboratory. We select $11.1 \pm 4.6$ candidate events, corresponding to a signal significance of $2.9 \sigma$, and obtain the branching fraction of $\left(1.14 \pm 0.47_{\text {(stat) }} \pm 0.17_{\text {(syst) }}\right) \times 10^{-7}$.

The 39th International Conference on High Energy Physics (ICHEP2018)

4-11 July, 2018

Seoul, Korea

* Speaker.

${ }^{\dagger}$ On behalf of the BABAR collaboration. 


\section{Introduction}

The discrepancy between the inclusive branching fraction of all $B$ meson decay modes with at least a couple of baryons in the final state, measured by ARGUS to be $(6.8 \pm 0.6) \%$ [1], and the sum of the measured exclusive baryonic decays of the $B$ meson which yields about 1\% [2] is still an unsolved puzzle and motivates further searches for yet unmeasured $B$-meson decays to baryons. The most relevant open issues concern the observed hierarchy of the branching fractions, due to resonant subchannels, and the threshold enhancement effect, observed as a decay rate increasing at the baryon-antibaryon invariant mass threshold. Providing a better understanding of these effects may allow progress in explaining the mechanism of hadronization into baryons. A previous search at $B A B A R$ has been performed for the purely baryonic four-body decay $\bar{B}^{0} \rightarrow \Lambda_{c}^{+} p \bar{p} \bar{p}$ [3], for which no signal events were observed. The upper limit on the branching fraction was computed to be $2.8 \times 10^{-6}$ at $90 \% \mathrm{CL}$. From this result, we estimate the expected branching fraction $\mathscr{B}(B \rightarrow$ $\left.p p \bar{p} \bar{p}^{1}\right) \sim 10^{-7}$, which would imply a few number of signal events at the available integrated luminosity at BABAR [4]. This calculation results by applying two scaling factors, one due to the Cabibbo suppression for the $b \rightarrow u$ decay, and one for the enlargement of the phase space, due to the lower proton mass with respect to the $\Lambda_{c}^{+}$.

\section{BABAR experiment and data set}

The analysed data set corresponds to $424 \mathrm{fb}^{-1}$ of $e^{+} e^{-}$collisions at the centre-of-mass (CM) energy of the $\Upsilon(4 S)$ resonance, $\sqrt{s}=10.58 \mathrm{GeV}$, collected with the BABAR detector, whose detailed description can be found in [5]. The tracking system consists of a five-layer double-sided silicon vertex tracker and a 40-layer multiwire drift chamber, both operating in the $1.5 \mathrm{~T}$ magnetic field of a superconducting solenoid, and responsible to provide the precise measurement of charged-particle momenta. The particle identification algorithms (PID) for protons, kaons and pions exploit the information on the specific energy loss measured by the tracking devices and on the Cherenkov angle mesurement, provided by the internally reflecting ring-imaging Cherenkov detector. A CsI(Tl) crystal electromagnetic calorimeter measures the energy deposit of particles and photons traversing it, which is then used for the identification of charged and neutral particles. Monte Carlo (MC) events corresponding to at least three times the data integrated luminosity are used to evaluate the signal efficiency, optimize the background rejection and model the signal shape.

\section{Analysis method}

The event reconstruction is performed by combining four oppositely charged tracks identified as protons and antiprotons and kinematically fitted to a common vertex, with a fit probability larger than $0.1 \%$. A loose selection is also applied to the kinematic variables $m_{\mathrm{ES}}=\sqrt{\left(E_{\text {beam }}^{*}\right)^{2}-\left(\overrightarrow{p_{\mathrm{B}}}{ }^{*}\right)^{2}}$ and $\Delta E=E_{\mathrm{B}}^{*}-E_{\text {beam }}^{*}[6]$, related to the momentum ${\overrightarrow{p_{\mathrm{B}}}}^{*}$ and the reconstructed energy $E_{\mathrm{B}}^{*}$ of the $B$-candidate and to the beam energy $E_{\text {beam }}^{*}$, in the CM reference frame $\left(m_{\mathrm{ES}}>5.2 \mathrm{GeV} / c^{2},|\Delta E|<\right.$ $0.2 \mathrm{GeV}$ ). The PID efficiency for protons of about $1 \mathrm{GeV} / c$ mean momentum is excellent (> 99\%) and mis-identification rates for wrongly assigning the proton identity to kaons and pions

\footnotetext{
${ }^{1}$ the charge conjugate is always implied throughout the article
} 
are lower than $1 \%$. The background is mainly combinatoric produced by real protons coming from continuum hadronization processes $\left(e^{+} e^{-} \rightarrow q \bar{q}\right)$, and it is rejected by imposing a signal-like selection on the output of a Boosted Decision Tree (BDT) algorithm. The BDT classifier exploits the following input variables: $\Delta E, \cos \theta_{\mathrm{B}}^{*}$, with $\theta_{\mathrm{B}}^{*}$ being the flight polar angle of the $B$ meson in the CM reference, and the event-shape variables $R_{2},\left|\cos \theta_{\mathrm{TH}}\right|$, which discriminate between the spherical shape of a signal event $\left(e^{+} e^{-} \rightarrow B \bar{B}\right)$ and a jet-like $q \bar{q}$ event. Respectively, $R_{2}$ consists in the ratio between the second and the zeroth Fox Wolfram moments [7] and $\theta_{\mathrm{TH}}$ is the angle between the Thrust axis [8] of the rest of the event evaluated in the CM frame and that of the $B$ candidate. The signal efficiency for the applied selection, computed on the signal MC sample, is $\varepsilon=0.207 \pm 0.005$. The associated uncertainty is systematic and takes into account the contributions from the PID and tracking efficiency, and from the BDT selection.

The signal shape is described by a Gaussian function, whose width and mean are fixed to the results obtained from the fit to the $m_{\mathrm{ES}}$ distribution on the signal $\mathrm{MC}$ sample. The combinatorial background shape is modeled by using an empirical ARGUS function [9], which depends on two parameters, the ARGUS cutoff and the ARGUS shape. The first is completely determined by the kinematics, being the endpoint of the $m_{\mathrm{ES}}$ distribution, and it is fixed to $5.289 \mathrm{GeV} / c^{2}$ in the final fit; the ARGUS shape is left floating and determined in the fit to the data, as well as the signal and the background yields.

\section{Results and systematic uncertainties}

The signal yield is extracted from an unbinned extended maximum likelihood fit to the $m_{\mathrm{ES}}$ distribution of the selected events in the range $5.2<m_{\mathrm{ES}}<5.3 \mathrm{GeV} / \mathrm{c}^{2}$ as shown in Figure 1, which results in $N_{\text {sig }}=11.1 \pm 4.6$ candidate events with a total significance of $2.9 \sigma$. The corresponding branching fraction is calculated $\mathscr{B}\left(B^{0} \rightarrow p p \bar{p} \bar{p}\right)=N_{\text {sig }} / \varepsilon \cdot N_{B \bar{B}}=\left(1.14 \pm 0.47_{\text {(stat) }} \pm 0.17_{\text {(syst) }}\right) \times$ $10^{-7}$, where the experimental inputs are $N_{\text {sig }}, \varepsilon$ and the number of $B$ meson pairs $N_{\mathrm{B} \overline{\mathrm{B}}}$. The largest contribution to the total uncertainty on the branching fraction comes from the low number of fitted signal events.

The systematic uncertainty on the branching fraction arises from three sources: the number of $B$-meson pairs $N_{\mathrm{B} \overline{\mathrm{B}}}$ [10], the fit procedure and the signal efficiency. The main contribution to the systematic uncertainty comes from the unknown dynamics of the decay model and it is assessed by using a weighting technique, based on the comparison of the momentum spectra of the decay products obtained with a pure phase space model, as assumed in the default signal MC simulation, and an alternative model, in which the decay proceeds through an intermediate spinless resonance. The contribution to the systematic uncertainty is estimated from the difference in the signal efficiency computed on weighted and unweighted signal MC samples and amounts to $14 \%$. The total relative systematic uncertainty of $15 \%$ is finally obtained by summing all the contributions in quadrature. The final result for the branching fraction is $\mathscr{B}(B \rightarrow p p \bar{p} \bar{p})=\left(1.14 \pm 0.47_{\text {(stat) }} \pm\right.$ $\left.0.17_{(\mathrm{sys})}\right) \times 10^{-7}$ and the $90 \%$ C.L. upper limit is also computed by generating pseudo-experiments which take into account the systematic variation and it yields $2.0 \times 10^{-7}$. 


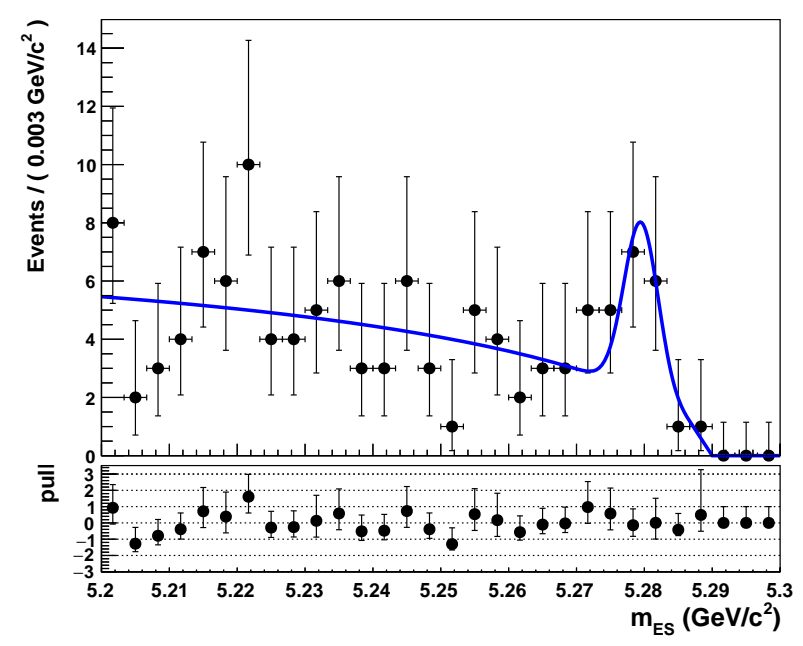

Figure 1: Fit to the data $m_{\mathrm{ES}}$ distribution (dots) in the interval $5.2<m_{\mathrm{ES}}<5.3 \mathrm{GeV} / \mathrm{c}^{2}$. The bottom plot shows the pull distribution, which is the bin-by-bin difference between the data and fitted distribution normalized by the corresponding statistical uncertainty from the fit.

\section{Conclusions}

In summary, we have performed a search for $B$ meson decays to the $p p \bar{p} \bar{p}$ final state [4], obtaining 11.1 signal events. The significance of the result, including both the statistical and systematic uncertainties, is 2.9 standard deviations. The branching fraction is measured to be $\mathscr{B}(B \rightarrow p p \bar{p} \bar{p})=\left(1.14 \pm 0.47_{\text {(stat) }} \pm 0.17_{\text {(sys) }}\right) \times 10^{-7}$. The corresponding $90 \%$ C.L. upper limit is $\mathscr{B}(B \rightarrow p p \bar{p} \bar{p})<2.0 \times 10^{-7}$. Our result can provide important input for QCD models of hadronization and improve understanding of the threshold enhancement effect.

\section{References}

[1] Albrecht H. et al., Zeitschrift für Physik C Particles and Fields, 56 (1992).

[2] C. Patrignani et al. [Particle Data Group], Chin. Phys. C 40, no. 10, 100001 (2016).

[3] J. P. Lees et al. [BaBar Collaboration], Phys. Rev. D 89, no. 7, 071102 (2014).

[4] J. P. Lees et al. [BaBar Collaboration], Phys. Rev. D,98, no.7, 071102 (2018).

[5] B. Aubert et al. [BaBar Collaboration], Nucl. Instrum. Meth. A 479, 1 (2002).

[6] A. J. Bevan et al. [BaBar and Belle Collaborations], Eur. Phys. J. C 74, 3026 (2014).

[7] G. C. Fox and S. Wolfram, Nucl. Phys. B 149, 413 (1979). Erratum: [Nucl. Phys. B 157, 543 (1979)].

[8] E. Farhi, Phys. Rev. Lett. 39, 1587 (1977).

[9] H. Albrecht et al., Phys. Lett. B, 241, 2 (1990).

[10] G. D. McGregor, arXiv:0812.1954 [hep-ex]. 